\title{
基于数能同传的 D2D 网络鲁棒资源分配算法
}

\author{
徐勇军 $1,2^{*} ，$ 杨蒙 ${ }^{1,2} ，$ 周继华 ${ }^{3} ，$ 陈前斌 1,2
}

\author{
1. 重庆邮电大学通信与信息工程学院, 重庆 400065 \\ 2. 移动通信技术重点实验室, 重庆 400065 \\ 3. 航天新通科技有限公司, 重庆 401332 \\ * 通信作者. E-mail: xuyj@cqupt.edu.cn
}

收稿日期: 2021-03-10；修回日期: 2021-04-25；接受日期: 2021-07-22；网络出版日期: 2022-10-09

国家自然科学基金 (批准号: 61601071, 62071078)、国家重点研发计划（批准号: 2019YFC1511300)、重庆市自然科学基金 (批准 号: cstc2019jcyjxfkxX0002) 和重庆市研究生科研创新项目 (批准号: CYS21294) 资助

\begin{abstract}
摘要为了提高终端直通 (device-to-device, D2D) 网络设备运行寿命和复杂电磁环境下的抗干扰能 力, 提出了一种基于数能同传的 $\mathrm{D} 2 \mathrm{D}$ 网络系统能效最大化的鲁棒资源分配算法. 考虑用户最小速率 约束、最小收集能量约束、功率分流和信道分配因子约束, 建立了联合优化传输功率、子信道和功率 分流因子的鲁棒能效资源分配模型。基于 Dinkelbach 方法和 Worst-case 方法, 将原 NP-hard 问题转 换为确定性的凸优化问题, 利用匹配理论和拉格朗日对偶理论获得解析解. 仿真结果表明所提算法具 有较好的鲁棒性和较高的能效.
\end{abstract}

关键词 D2D 通信, 无线信息与功率同传技术, 匹配理论, 能效, 鲁棒资源分配

\section{1 引言}

终端直通 (device-to-device, D2D) 技术允许设备与设备之间进行直接通信, 具有高容量、高能效 的特点, 被认为是 $5 \mathrm{G} / \mathrm{B} 5 \mathrm{G}$ 的核心技术之一 ${ }^{[1]}$. 随着设备数量的不断增加, 如何有效提高系统能效、 延长设备使用寿命、实现绿色通信是未来 D2D 网络的一个关键问题 ${ }^{[2]}$.

能量收集 (energy harvesting, EH) 技术通过从环境中收集能量, 能够有效延长能量受限无线网络的 运行寿命 ${ }^{[3]}$. 由于无线射频信号广泛应用于无线信息传输中, 无线信息与功率同传技术 (simultaneous wireless information and power transfer, SWIPT), 即数能同传技术, 近年来受到了广泛关注 ${ }^{[4]}$. SWIPT 技术通过时间切换或能量分流机制允许无线设备在接收无线信息的同时收集环境中的电磁能量, 能够 有效提高数据传输效率与能量利用率. 因此, 如何实现最优时间切换或能量分流是实现基于 SWIPT 的无线网络系统能效最大化的关键所在 ${ }^{[5]}$.

\footnotetext{
引用格式: 徐勇军, 杨蒙, 周继华, 等. 基于数能同传的 D2D 网络鲁棒资源分配算法. 中国科学: 信息科学, 2022, 52: 1883-1899, doi: 10.1360/SSI-2021-0086

Xu Y J, Yang M, Zhou J H, et al. Robust resource allocation algorithm in SWIPT-based D2D networks (in Chinese). Sci Sin Inform, 2022, 52: 1883-1899, doi: 10.1360/SSI-2021-0086
} 
因此, 在 D2D 网络中融入 SWIPT 技术 (即, 基于数能同传的 D2D 网络) 可以实现无线信息与电 磁能量同时传输, 有效解决 D2D 设备能量短缺问题 ${ }^{1}$. 为了提高数能同传 $\mathrm{D} 2 \mathrm{D}$ 网络能量利用率 ${ }^{[6]}$, 对 资源分配算法的研究受到广泛关注. 针对灾难场景下的数能同传 D2D 网络, 文献 [7] 提出了一种基于 时间切换协议的能效最大化资源分配算法. 文献 [8] 基于全双工的中继 D2D 网络, 设计了一种在信息 传输和能量传输之间进行权衡的策略以最大限度的提高 $\mathrm{D} 2 \mathrm{D}$ 链路的数据速率. 基于博亦论, 文献 [9] 提出了一种功率分流协议的能效最大化资源分配算法. 为了提高频谱效率, 文献 [10] 通过联合优化传 输功率和资源块 (resource block, RB), 使得系统的能效最大化. 考虑蜂窝用户 (cellular user, CU) 和 D2D 用户 (D2D user, DU) 最小速率约束, 文献 [11] 通过对传输功率、功率分流因子联合优化, 实现系 统能效最大化. 针对基于非正交多址接入的数能同传 D2D 网络, 文献 [12] 在保证蜂窝用户最小速率 需求的情况下最大化 $\mathrm{D} 2 \mathrm{D}$ 用户的和速率. 上述工作都假设信道状态信息可以完美获得, 该假设在实际 场景中过于理想. 考虑非完美信道状态信息, 文献 [13] 针对数能同传的 D2D 网络提出了一种速率最 大化的鲁棒资源分配算法. 文献 [14] 研究了基于非正交多址接入的数能同传 D2D 网络鲁棒资源分配 算法.

上述大部分工作都假设信道状态信息可以精确获得, 没有考虑信道不确定性的影响, 且目标函数 只考虑了 D2D 用户或蜂窝用户的性能优化, 没有考虑整体系统性能. 虽然文献 [14] 研究了信道不 确定性下的鲁棒和速率资源分配问题, 但没有考虑系统整体能效, 同时忽略了能量收集对系统性能的 影响.

为克服信道不确定性引起的用户中断, 同时提高系统能量利用率, 本文研究基于数能同传的 $\mathrm{D} 2 \mathrm{D}$ 网络系统能效最大化鲁棒资源分配问题. 主要贡献如下:

(1) 针对基于数能同传的多用户 D2D 网络上行传输场景. 考虑蜂窝用户和 D2D 用户的最小速率 约束、最小收集能量约束、信道分配约束、能量分流因子约束, 以及最大发射功率约束, 基于有界信 道不确定性模型, 建立了一个联合功率分配、信道分配和功率分流因子优化的鲁棒能效资源分配模型. 该模型是一个含整数变量、多变量耦合、非凸优化问题, 很难直接获得其解析解.

(2) 基于 Worst-case 方法将鲁棒优化问题转化为确定优化问题, 利用匹配理论实现信道分配; 同 时, 利用 Dinkelbach 方法将上述优化问题转化为非分式形式, 并利用连续凸近似、指数变换和拉格朗 日对偶理论获得解析解.

(3) 仿真结果表明, 与现有算法对比, 本文算法具有较好的收玫性、能效和鲁棒性.

\section{2 系统模型及问题描述}

考虑基于数能同传的 D2D 网络上行传输场景, 如图 1 所示. $M$ 个蜂窝用户采用正交频分多址接 入方式实现上行传输. $N$ 个 D $2 \mathrm{D}$ 用户对具有数能同传功能, 且采用下垫式频谱共享方式复用蜂窝用 户的信道资源. 每个子信道上同一时间只能允许一对 D2D 用户对接入. 定义蜂窝用户和 D2D 用户集 合分别为 $\mathcal{M}=\{1,2, \ldots, M\}, \forall m \in \mathcal{M}$ 和 $\mathcal{N}=\{1,2, \ldots, N\}, \forall n \in \mathcal{N}$. 假设每个子信道服从块衰落.

考虑 D2D 用户对蜂窝用户的干扰, 第 $m$ 个蜂窝用户上行传输的信干噪比为

$$
\gamma_{m}^{\mathrm{C}}=\frac{P_{m} g_{m, 0}}{\sigma^{2}+\sum_{n=1}^{N} x_{n, m} p_{n} h_{n, 0}},
$$

1) 能量收集技术允许 D2D 设备收集周围环境的射频能源, 从而可以实现电池容量受限 D2D 设备的无线充电功 能. 因此能量收集技术与 $\mathrm{D} 2 \mathrm{D}$ 通信网络结合可以在提高传输速率的同时也能够提高能量利用率. 


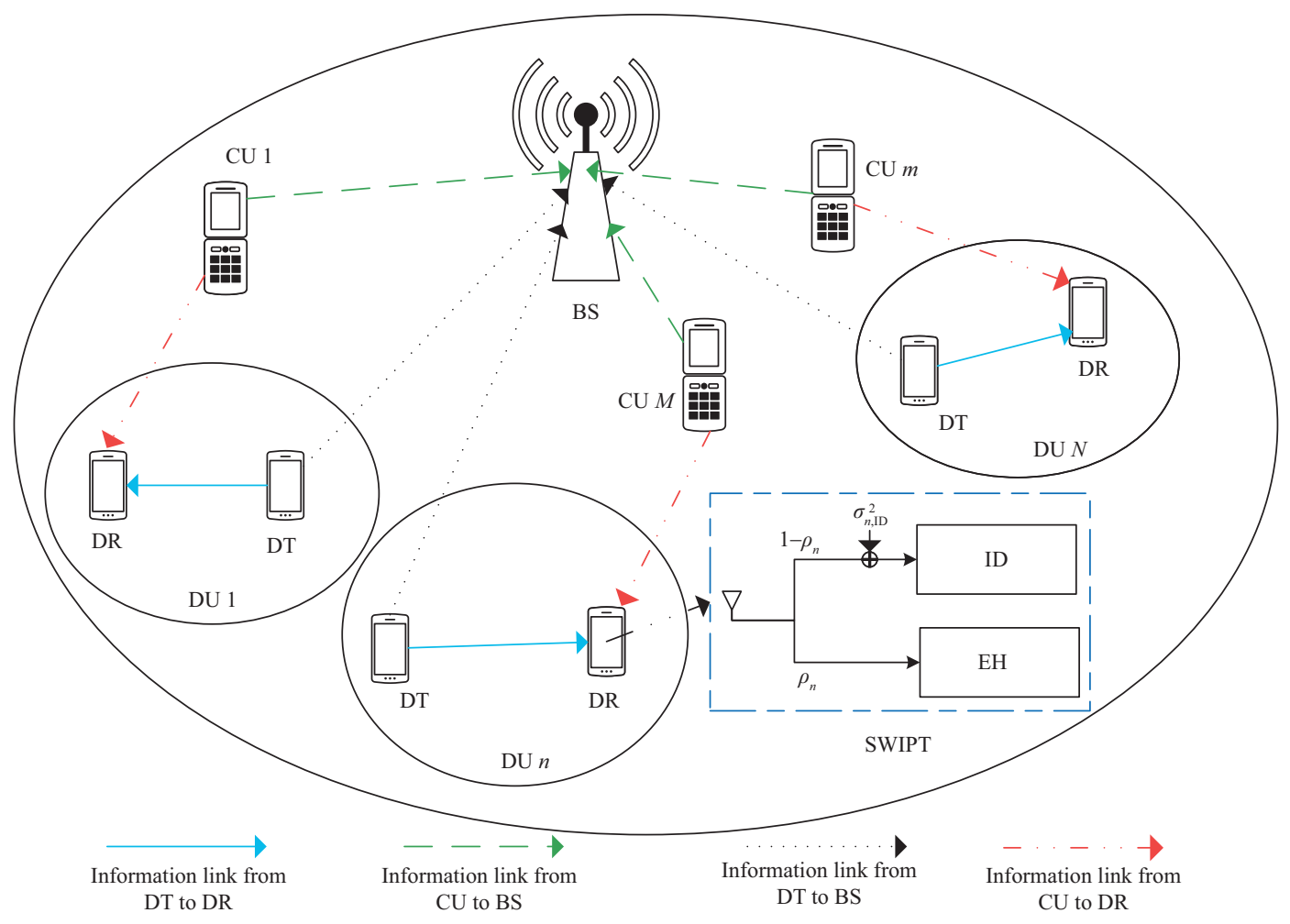

图 1 (网络版彩图) 基于数能同传的 D2D 网络系统模型

Figure 1 (Color online) A SWIPT-based D2D network

其中, $P_{m}$ 是第 $m$ 个蜂窝用户到基站 (base station, BS) 的发射功率; $g_{m, 0}$ 是第 $m$ 个蜂窝用户到基站的 信道增益; $p_{n}$ 是第 $n$ 对 D2D 用户发射机 (D2D user transmitter, DT) 的传输功率; $h_{n, 0}$ 是第 $n$ 对 D2D 用户发射机到基站的信道增益; $\sigma^{2}$ 是基站处的背景噪声功率. $x_{n, m}$ 是信道分配因子. $x_{n, m}=1$ 表示第 $n$ 对 D2D 用户复用第 $m$ 个蜂窝用户的子信道, 否则 $x_{n, m}=0$, 且满足 $\sum_{m} x_{n, m} \leqslant 1, \sum_{n} x_{n, m} \leqslant 1$, $x_{n, m}=\{0,1\}$.

考虑蜂窝用户对 $\mathrm{D} 2 \mathrm{D}$ 用户的共道干扰, 在不考虑能量收集的情况下, 第 $n$ 对 $\mathrm{D} 2 \mathrm{D}$ 用户接收机 (D2D user receiver, DR) 的信干噪比为

$$
\gamma_{n}^{\mathrm{D}}=\frac{p_{n} h_{n, n}}{\sigma_{n}^{2}+\sum_{m=1}^{M} x_{n, m} P_{m} g_{m, n}},
$$

其中, $h_{n, n}$ 是第 $n$ 对 D2D 用户发射机到接收机的信道增益; $g_{m, n}$ 是蜂窝用户 $m$ 到第 $n$ 对 D2D 用户 接收机的信道增益; $\sigma_{n}^{2}$ 是第 $n$ 对 D2D 用户接收机处的背景噪声功率. $\sum_{m=1}^{M} x_{n, m} P_{m} g_{m, n}$ 表示蜂窝用 户对第 $n$ 对 $\mathrm{D} 2 \mathrm{D}$ 用户的干扰功率.

基于功率分流模型 ${ }^{[3]}$, 式 (2) 的信干噪比可重新描述为

$$
\gamma_{n}^{\mathrm{DS}}=\frac{\left(1-\rho_{n}\right) p_{n} h_{n, n}}{\sigma_{n, \mathrm{ID}}^{2}+\left(1-\rho_{n}\right)\left(\sigma_{n}^{2}+\sum_{m=1}^{M} x_{n, m} P_{m} g_{m, n}\right)},
$$

其中, $\rho_{n} \in[0,1]$ 表示第 $n$ 对 $\mathrm{D} 2 \mathrm{D}$ 用户的功率分流因子 ${ }^{[11]}, \sigma_{n, \mathrm{ID}}^{2}$ 是第 $n$ 对 $\mathrm{D} 2 \mathrm{D}$ 用户接收机信息解 
码 (information decoding, ID) 过程引入的噪声功率. 因此, 第 $n$ 对 D2D 用户接收机收集的能量为

$$
E_{n}^{\mathrm{D}}=\varsigma \rho_{n}\left(\sigma_{n}^{2}+p_{n} h_{n, n}+\sum_{m=1}^{M} x_{n, m} P_{m} g_{m, n}\right),
$$

其中, $\varsigma \in[0,1]$ 表示能量收集效率因子 ${ }^{[11]}$.

由以上分析, 根据香农 (Shannon) 定理, 蜂窝用户速率、D2D 用户速率和系统总速率可以表示为

$$
\begin{aligned}
& R_{m}^{\mathrm{C}}=\log _{2}\left(1+\gamma_{m}^{\mathrm{C}}\right), \\
& R_{n}^{\mathrm{DS}}=\log _{2}\left(1+\gamma_{n}^{\mathrm{DS}}\right), \\
& R^{\mathrm{sum}}=\sum_{m=1}^{M} R_{m}^{\mathrm{C}}+\sum_{n=1}^{N} R_{n}^{\mathrm{DS}} .
\end{aligned}
$$

系统消耗的总能量为

$$
E^{\text {sum }}=\sum_{m=1}^{M} P_{m}+P_{\text {cir }}+\sum_{n=1}^{N} p_{n}+N p_{\text {cir }}-\sum_{n=1}^{N} E_{n}^{\mathrm{D}},
$$

其中, $P_{\text {cir }}$ 和 $p_{\text {cir }}$ 分别表示系统里面所有蜂窝用户和任意一对 D2D 链路的电路消耗. 为了保证公平性, 假设每对 D2D 链路的电路消耗相等. 为了保护蜂窝用户的通信质量, 考虑 D2D 用户最小传输速率和 能量收集约束, 基于系统总能效最大化的资源分配模型可以描述为

$$
\begin{aligned}
& \max _{P_{m}, p_{n}, \rho_{n}, x_{n, m}} \frac{R^{\text {sum }}}{E^{\text {sum }}} \\
& \text { s.t. } \mathrm{C}_{1}: 0 \leqslant \rho_{n} \leqslant 1, \quad \forall n, \\
& \mathrm{C}_{2}: R_{n}^{\mathrm{DS}} \geqslant R_{n}^{\mathrm{D}, \min }, \quad \forall n, \\
& \mathrm{C}_{3}: R_{m}^{\mathrm{C}} \geqslant R_{m}^{\mathrm{C}, \min }, \quad \forall m, \\
& \mathrm{C}_{4}: 0 \leqslant p_{n} \leqslant p_{n}^{\max }, \quad \forall n, \\
& \mathrm{C}_{5}: 0 \leqslant P_{m} \leqslant P_{m}^{\max }, \quad \forall m, \\
& \mathrm{C}_{6}: E_{n}^{\mathrm{D}} \geqslant E_{n}^{\min }, \quad \forall n, \\
& \mathrm{C}_{7}: \sum_{m=1}^{M} x_{n, m} \leqslant 1, \quad \sum_{n=1}^{N} x_{n, m} \leqslant 1, \quad x_{n, m}=\{0,1\},
\end{aligned}
$$

其中, $R_{n}^{\mathrm{D}, \min }$ 表示第 $n$ 对 $\mathrm{D} 2 \mathrm{D}$ 用户接收机的最小速率门限, $R_{m}^{\mathrm{C}, m i n}$ 表示第 $m$ 个蜂窝用户的最小速 率门限, $p_{n}^{\max }$ 是第 $n$ 对 $\mathrm{D} 2 \mathrm{D}$ 用户发射机的最大发射功率, $P_{m}^{\max }$ 是第 $m$ 个蜂窝用户的最大发射功率, $E_{n}^{\min }$ 表示激活第 $n$ 对 $\mathrm{D} 2 \mathrm{D}$ 用户接收机能量收集电路的最小能量阈值, 该值可以保证接收机收集的 能量始终可以达到能量收集电路正常工作所需要的最小能量 ${ }^{[11]} . \mathrm{C}_{1}$ 是功率分流因子约束; $\mathrm{C}_{2}$ 是每对 D2D 用户接收机的最小速率约束; $\mathrm{C}_{3}$ 是每个蜂窝用户的最小速率约束; $\mathrm{C}_{4}$ 是每对 $\mathrm{D} 2 \mathrm{D}$ 用户对的最 大发射功率约束; $\mathrm{C}_{5}$ 是每个蜂窝用户的最大发射功率约束; $\mathrm{C}_{6}$ 是每对 $\mathrm{D} 2 \mathrm{D}$ 用户接收机的最小能量收 集约束, 收集的能量要大于能量收集电路的最小激活能量; $\mathrm{C}_{7}$ 是子信道分配约束. 


\section{3 鲁棒资源分配算法设计}

\section{1 鲁棒问题描述与转换}

由于信道估计误差的影响, 导致完美的信道状态信息很难准确得到, 基于加性不确定性模型 ${ }^{[15]}$, 问题 (9) 中信道不确定性可建模为

$$
\left\{\begin{array}{l}
h_{n, n}=\bar{h}_{n, n}+\Delta h_{n, n}, \Delta h_{n, n} \in \mathcal{R}_{h}, \forall n \\
g_{m, n}=\bar{g}_{m, n}+\Delta g_{m, n}, \Delta g_{m, n} \in \mathcal{R}_{g}, \forall m, n \\
h_{n, 0}=\bar{h}_{n, 0}+\Delta h_{n, 0}, \Delta h_{n, 0} \in \mathcal{R}_{h_{0}}, \forall n
\end{array}\right.
$$

其中, $\bar{h}_{n, n}, \bar{g}_{m, n}$ 和 $\bar{h}_{n, 0}$ 是信道估计值, $\Delta h_{n, n}, \Delta g_{m, n}$ 和 $\Delta h_{n, 0}$ 是相应的估计误差. $\mathcal{R}_{h}, \mathcal{R}_{g}$ 和 $\mathcal{R}_{h_{0}}$ 表 示不确定性集合. 根据椭球不确定性集合 [16], 不确定性集合可定义为

$$
\left\{\begin{array}{l}
\mathcal{R}_{h}=\left\{\left.\Delta h_{n, n}\left|\sum_{k=1}^{N}\right| \Delta h_{n, n}\right|^{2} \leqslant \varepsilon_{n}^{2}\right\}, \\
\mathcal{R}_{g}=\left\{\left.\Delta g_{m, n}\left|\sum_{m=1}^{M}\right| \Delta g_{m, n}\right|^{2} \leqslant v_{n}^{2}\right\}, \\
\mathcal{R}_{h_{0}}=\left\{\left.\Delta h_{n, 0}\left|\sum_{n=1}^{N}\right| \Delta h_{n, 0}\right|^{2} \leqslant \xi_{0}^{2}\right\},
\end{array}\right.
$$

其中, $\varepsilon_{n} \geqslant 0, v_{n} \geqslant 0$ 和 $\xi_{0} \geqslant 0$ 表示不确定性上界 [17]. 结合式 (9) 和 (11), 得到如下鲁棒资源分配 问题:

$$
\begin{aligned}
& \max _{P_{m}, p_{n}, \rho_{n}, x_{n, m}} \frac{\min _{\Delta h_{n, 0}, \Delta h_{n, n}, \Delta g_{m, n}} R^{\operatorname{sum}}\left(\Delta h_{n, 0}, \Delta h_{n, n}, \Delta g_{m, n}\right)}{\max _{\Delta h_{n, n}, \Delta g_{m, n}} E^{\operatorname{sum}}\left(\Delta h_{n, n}, \Delta g_{m, n}\right)} \\
& \text { s.t. } \mathrm{C}_{1}, \mathrm{C}_{4}, \mathrm{C}_{5}, \mathrm{C}_{7} \text {, } \\
& \overline{\mathrm{C}}_{2}: \min _{\Delta h_{n, n}, \Delta g_{m, n}} R_{n}^{\mathrm{DS}}\left(\Delta h_{n, n}, \Delta g_{m, n}\right) \geqslant R_{n}^{\mathrm{D}, \min }, \quad \forall n, \\
& \overline{\mathrm{C}}_{3}: \min _{\Delta h_{n, 0}} R_{m}^{\mathrm{C}}\left(\Delta h_{n, 0}\right) \geqslant R_{m}^{\mathrm{C}, \min }, \quad \forall m, \\
& \overline{\mathrm{C}}_{6}: \min _{\Delta h_{n, n}, \Delta g_{m, n}} E_{n}^{\mathrm{D}}\left(\Delta h_{n, n}, \Delta g_{m, n}\right) \geqslant E_{n}^{\min }, \quad \forall n, \\
& \mathrm{C}_{8}: \mathcal{R}_{h}=\left\{\left.\Delta h_{n, n}\left|\sum_{k=1}^{N}\right| \Delta h_{n, n}\right|^{2} \leqslant \varepsilon_{n}^{2}\right\} \\
& \mathrm{C}_{9}: \mathcal{R}_{g}=\left\{\left.\Delta g_{m, n}\left|\sum_{m=1}^{M}\right| \Delta g_{m, n}\right|^{2} \leqslant v_{n}^{2}\right\} \\
& \mathrm{C}_{10}: \mathcal{R}_{h_{0}}=\left\{\left.\Delta h_{n, 0}\left|\sum_{n=1}^{N}\right| \Delta h_{n, 0}\right|^{2} \leqslant \xi_{0}^{2}\right\} .
\end{aligned}
$$

根据三角不等式, 可得

$$
\begin{aligned}
& \left|p_{n} \bar{h}_{n, 0}\right|-\left|p_{n} \Delta h_{n, 0}\right| \leqslant\left|p_{n}\left(\bar{h}_{n, 0}+\Delta h_{n, 0}\right)\right| \leqslant\left|p_{n} \bar{h}_{n, 0}\right|+\left|p_{n} \Delta h_{n, 0}\right|, \\
& \left|P_{m} \bar{g}_{m, n}\right|-\left|P_{m} \Delta g_{m, n}\right| \leqslant\left|P_{m}\left(\bar{g}_{m, n}+\Delta g_{m, n}\right)\right| \leqslant\left|P_{m} \bar{g}_{m, n}\right|+\left|P_{m} \Delta g_{m, n}\right| .
\end{aligned}
$$


根据柯西 - 施瓦茨 (Cauchy-Schwarz) 不等式 [15], 可得

$$
\begin{aligned}
& \sum_{n=1}^{N} p_{n} \Delta h_{n, 0} \leqslant \sqrt{\sum_{n=1}^{N}\left(\Delta h_{n, 0}\right)^{2}} \sqrt{\sum_{n=1}^{N}\left(p_{n}\right)^{2}} \leqslant \xi_{0} \sum_{n=1}^{N} p_{n}, \\
& \sum_{m=1}^{M} P_{m} \Delta g_{m, n} \leqslant \sqrt{\sum_{m=1}^{M}\left(\Delta g_{m, n}\right)^{2}} \sqrt{\sum_{m=1}^{M}\left(P_{m}\right)^{2}} \leqslant v_{n} \sum_{m=1}^{M} P_{m} .
\end{aligned}
$$

根据定义 $\sum_{k=1}^{N}\left|\Delta h_{n, n}\right|^{2} \leqslant \varepsilon_{n}^{2}$, 考虑信道不确定性平均估计误差, 则有 $\left|\Delta h_{n, n}\right|^{2} \leqslant \varepsilon_{n}^{2} / N$. 因此

$$
\min _{\Delta h_{n, n}}\left\{p_{n} h_{n, n}\left(\Delta h_{n, n}\right)\right\}=p_{n}\left(\bar{h}_{n, n}-\bar{\varepsilon}_{n}\right),
$$

其中, $\bar{\varepsilon}_{n}=\varepsilon_{n} / \sqrt{N}$. 将式 (13)-(17) 代入 (12), 可得

$$
\begin{aligned}
& \max _{P_{m}, p_{n}, \rho_{n}, x_{n}, m} \frac{\sum_{m=1}^{M} \log _{2}\left(1+\bar{\gamma}_{m}^{\mathrm{C}}\right)+\sum_{n=1}^{N} \log _{2}\left(1+\bar{\gamma}_{n}^{\mathrm{DS}}\right)}{\sum_{m=1}^{M} P_{m}+P_{\text {cir }}+\sum_{n=1}^{N} p_{n}+N p_{\text {cir }}-\sum_{n=1}^{N} \bar{E}_{n}^{\mathrm{D}}} \\
& \text { s.t. } \mathrm{C}_{1}, \mathrm{C}_{4}, \mathrm{C}_{5}, \mathrm{C}_{7}, \\
& \quad \tilde{\mathrm{C}}_{2}: \log _{2}\left(1+\bar{\gamma}_{n}^{\mathrm{DS}}\right) \geqslant R_{n}^{\mathrm{D}, \min }, \quad \forall n, \\
& \quad \tilde{\mathrm{C}}_{3}: \log _{2}\left(1+\bar{\gamma}_{m}^{\mathrm{C}}\right) \geqslant R_{m}^{\mathrm{C}, \min }, \quad \forall m, \\
& \quad \tilde{\mathrm{C}}_{6}: \bar{E}_{n}^{\mathrm{D}} \geqslant E_{n}^{\min }, \quad \forall n,
\end{aligned}
$$

其中,

$$
\begin{aligned}
\bar{\gamma}_{m}^{\mathrm{C}} & =\frac{P_{m} g_{m, 0}}{\sigma^{2}+\sum_{n=1}^{N} x_{n, m} p_{n}\left(\bar{h}_{n, 0}+\xi_{0}\right)}, \\
\bar{\gamma}_{n}^{\mathrm{DS}} & =\frac{\left(1-\rho_{n}\right) p_{n}\left(\bar{h}_{n, n}-\bar{\varepsilon}_{n}\right)}{\sigma_{n, \mathrm{ID}}^{2}+\left(1-\rho_{n}\right)\left(\sigma_{n}^{2}+\sum_{m=1}^{M} x_{n, m} P_{m}\left(\bar{g}_{m, n}+\bar{v}_{n}\right)\right)}, \\
\bar{E}_{n}^{\mathrm{D}} & =\varsigma \rho_{n}\left(\sigma_{n}^{2}+p_{n}\left(\bar{h}_{n, n}-\bar{\varepsilon}_{n}\right)+\sum_{m=1}^{M} x_{n, m} P_{m}\left(\bar{g}_{m, n}-\bar{v}_{n}\right)\right) .
\end{aligned}
$$

问题 (18) 是一个非凸、确定性的优化问题. 根据 Dinkelbach 方法 ${ }^{[14]}$, 式 (18) 中的目标函数可等 价为

$$
f(\eta)=\sum_{m=1}^{M} \log _{2}\left(1+\bar{\gamma}_{m}^{\mathrm{C}}\right)+\sum_{n=1}^{N} \log _{2}\left(1+\bar{\gamma}_{n}^{\mathrm{DS}}\right)-\eta\left(\sum_{m=1}^{M} P_{m}+P_{\text {cir }}+\sum_{n=1}^{N} p_{n}+N p_{\text {cir }}-\sum_{n=1}^{N} \bar{E}_{n}^{\mathrm{D}}\right),
$$

其中, $\eta$ 是一个非负的辅助变量, 表示整个系统的能效 ${ }^{[14]}$.

由于数据速率存在变量耦合, $\tilde{\mathrm{C}}_{2}$ 和 $\tilde{\mathrm{C}}_{3}$ 是非凸的. 为了解决该问题, 基于连续凸近似 ${ }^{[17]}$, 则有

$$
\begin{aligned}
& \log _{2}\left(1+\bar{\gamma}_{m}^{\mathrm{C}}\right) \geqslant a_{m} \log _{2}\left(\bar{\gamma}_{m}^{\mathrm{C}}\right)+b_{m}, \\
& \log _{2}\left(1+\bar{\gamma}_{n}^{\mathrm{DS}}\right) \geqslant c_{n} \log _{2}\left(\bar{\gamma}_{n}^{\mathrm{DS}}\right)+d_{n}
\end{aligned}
$$

其中,

$$
a_{m}=\widehat{\gamma}_{m}^{\mathrm{C}} /\left(1+\widehat{\gamma}_{m}^{\mathrm{C}}\right)
$$

1888 


$$
\begin{aligned}
& c_{n}=\widehat{\gamma}_{n}^{\mathrm{DS}} /\left(1+\widehat{\gamma}_{n}^{\mathrm{DS}}\right), \\
& b_{m}=\log _{2}\left(1+\widehat{\gamma}_{m}^{\mathrm{C}}\right)-a_{m} \log _{2}\left(\widehat{\gamma}_{m}^{\mathrm{C}}\right), \\
& d_{n}=\log _{2}\left(1+\widehat{\gamma}_{n}^{\mathrm{DS}}\right)-c_{n} \log _{2}\left(\widehat{\gamma}_{n}^{\mathrm{DS}}\right) .
\end{aligned}
$$

当 $\bar{\gamma}_{m}^{\mathrm{C}}=\widehat{\gamma}_{m}^{\mathrm{C}}$ 和 $\bar{\gamma}_{n}^{\mathrm{DS}}=\widehat{\gamma}_{n}^{\mathrm{DS}}$, 式 (23) 和 (24) 等号成立, 则有

$$
\begin{aligned}
& \bar{R}_{m}^{\mathrm{C}}=a_{m} \log _{2}\left(\bar{\gamma}_{m}^{\mathrm{C}}\right)+b_{m} \geqslant \tilde{R}_{m}^{\mathrm{C}}, \\
& \bar{R}_{n}^{\mathrm{DS}}=c_{n} \log _{2}\left(\bar{\gamma}_{n}^{\mathrm{DS}}\right)+d_{n} \geqslant \tilde{R}_{n}^{\mathrm{DS}},
\end{aligned}
$$

其中,

$$
\begin{aligned}
& \tilde{R}_{m}^{\mathrm{C}}=a_{m} \log _{2}\left(P_{m} g_{m, 0} / z_{m}\right)+b_{m}, \\
& z_{m} \geqslant \sigma^{2}+\sum_{n=1}^{N} x_{n, m} p_{n}\left(\bar{h}_{n, 0}+\xi_{0}\right), \\
& \tilde{R}_{n}^{\mathrm{DS}}=c_{n} \log _{2}\left(\left(1-\rho_{n}\right) p_{n}\left(\bar{h}_{n, n}-\bar{\varepsilon}_{n}\right) / q_{n}\right)+d_{n}, \\
& q_{n} \geqslant \sigma_{n, \mathrm{ID}}^{2}+\left(1-\rho_{n}\right)\left(\sigma_{n}^{2}+\sum_{m=1}^{M} x_{n, m} P_{m}\left(\bar{g}_{m, n}+v_{n}\right)\right) .
\end{aligned}
$$

根据式 (23)-(34), 问题 (18) 可以转换为如下优化问题:

$$
\begin{aligned}
& \max _{P_{m}, p_{n}, \rho_{n}, x_{n, m}, \eta, q_{n}, z_{m}} \sum_{m=1}^{M} \tilde{R}_{m}^{\mathrm{C}}+\sum_{n=1}^{N} \tilde{R}_{n}^{\mathrm{DS}}-\eta\left\{\sum_{m=1}^{M} P_{m}+P_{\text {cir }}+\sum_{n=1}^{N} p_{n}+N p_{\text {cir }}-\sum_{n=1}^{N} \bar{E}_{n}^{\mathrm{D}}\right\} \\
& \text { s.t. } \mathrm{C}_{1}, \mathrm{C}_{4}, \mathrm{C}_{5}, \tilde{\mathrm{C}}_{6}, \mathrm{C}_{7} \text {, } \\
& \widehat{\mathrm{C}}_{2}: \tilde{R}_{n}^{\mathrm{DS}} \geqslant R_{n}^{\mathrm{D}, \min }, \quad \forall n, \\
& \widehat{\mathrm{C}}_{3}: \tilde{R}_{m}^{\mathrm{C}} \geqslant R_{m}^{\mathrm{C}, \mathrm{min}}, \quad \forall m \text {, } \\
& \mathrm{C}_{11}: z_{m} \geqslant \sigma^{2}+\sum_{n=1}^{N} x_{n, m} p_{n}\left(\bar{h}_{n, 0}+\xi_{0}\right), \quad \forall m, \\
& \mathrm{C}_{12}: q_{n} \geqslant \sigma_{n, \mathrm{ID}}^{2}+\left(1-\rho_{n}\right)\left(\sigma_{n}^{2}+\sum_{m=1}^{M} x_{n, m} P_{m}\left(\bar{g}_{m, n}+v_{n}\right)\right), \quad \forall n \text {. }
\end{aligned}
$$

由于二进制变量 $x_{n, m}$ 和其他变量存在耦合关系, 问题 (35) 仍然是一个非凸优化问题. 为了解决 该问题, 利用匹配算法 ${ }^{[18]}$ 确定 $x_{n, m}$ 的值, 然后对剩下的凸优化问题进行求解.

\section{2 匹配算法设计}

在匹配算法设计部分, 本文针对给定蜂窝用户和 D2D 用户发射机传输功率以及分流因子的情况 下, 研究蜂窝用户和 D2D 用户对的匹配问题.

定义1 定义 $u$ 表示一对一匹配 $\mathcal{N} \otimes \mathcal{M}$ 的子集, 且满足 $|u(n)|=1$ 和 $|u(m)| \leqslant 1 .|u(n)|=1$ 保 证了第 $n$ 对 D2D 用户能匹配一个子信道, $|u(m)| \leqslant 1$ 保证了蜂窝用户 $m$ 所占用的子信道最多可以被 一对 D2D 用户匹配. 对于 $\forall n \in \mathcal{N}, \forall m \in \mathcal{M}$, 当且仅当 $u(n)=m$ 且 $u(m)=n$ 时, $x_{n, m}=1$. 
定义2 定义一个匹配集合 $u$, 两对 D2D 用户标号分别为 $n$ 和 $n^{\prime}$, 两个蜂窝用户标号分别为 $m$ 和 $m^{\prime}$, 且满足 $(n, m),\left(n^{\prime}, m^{\prime}\right) \in u$, 则交换 (swap) 匹配为 $u_{n}^{n^{\prime}}=u \backslash\left\{(n, m),\left(n^{\prime}, m^{\prime}\right)\right\} \cup\left\{\left(n, m^{\prime}\right),\left(n^{\prime}, m\right)\right\}$. 在交换匹配中, 两对 D2D 交换其匹配的蜂窝用户子信道, 其他 D2D 用户不变.

定义 3 定义一个匹配集合 $u$, 当且仅当 $\forall x \in\left\{n, n^{\prime}, u(n), u\left(n^{\prime}\right)\right\}, \Phi_{x}\left(u_{n}^{n^{\prime}}(x)\right) \geqslant \Phi_{x}(u(x))$ 和 $\exists x \in$ $\left\{n, n^{\prime}, u(n), u\left(n^{\prime}\right)\right\}, \Phi_{x}\left(u_{n}^{n^{\prime}}(x)\right)>\Phi_{x}(u(x))$ 成立时, 则两对 $\mathrm{D} 2 \mathrm{D}\left(n, n^{\prime}\right)$ 是匹配堵塞对 (swap-blocking pair). 其中, $\Phi_{x}(\cdot)$ 表示效用函数, 即在匹配堵塞对交换其匹配对象之后, 所有参与者的效益不能减少, 并且在匹配堵塞对交换其匹配对象之后, 至少有一个参与者的效益是提高的, 避免了相同效益的匹配 对反复交换.

定义 4 当所考虑的网络中不存在 $\mathrm{D} 2 \mathrm{D}$ 匹配堵塞对时, 网络是双向稳定的.

假设 $\mathrm{D} 2 \mathrm{D}$ 用户能够对子信道进行接入偏好排序, 则第 $n$ 对 D2D 用户的效用函数 ${ }^{[19]}$ 可以定义为

$$
\Phi_{n}=R_{n}^{\mathrm{DS}}-\kappa,
$$

其中, $\kappa$ 表示接入蜂窝用户子信道的代价. 同理, 第 $m$ 个蜂窝用户的效用函数可定义为

$$
\Phi_{m}=\kappa-R_{m}^{\mathrm{C}} \text {. }
$$

因此, D2D 用户与蜂窝用户子信道的匹配算法如算法 1 所示.

算法 1 的稳定性分析: 根据定义 3 , 只有当网络中存在 D2D 用户匹配堵塞对时, 才会发生交换, 且 交换之后参与交换的用户效益增加, 所以总的效益是严格递增的, 网络是稳定的. 如果网络中还存在 D2D 用户匹配堵塞对, 就还会进行交换, 此时网络未达到稳定.

\section{4 分流因子与功率分配的联合优化}

\section{1 分流因子优化子问题求解}

因为 $\rho_{n}$ 与 $\mathrm{C}_{1}, \widehat{\mathrm{C}}_{2}, \tilde{\mathrm{C}}_{6}$ 和 $\mathrm{C}_{12}$ 有关, 基于交替优化思想, 问题 (35) 可以分解为只含有 $\rho_{n}$ 的子问 题和其他变量的子问题. 定义 $\rho_{n}=e^{\rho_{n}^{\prime}}$, 问题 (35) 可重新描述为

$$
\begin{aligned}
& \max _{\rho_{n}^{\prime}} \sum_{m=1}^{M}\left\{a_{m} \log _{2}\left(P_{m} g_{m, 0} z_{m}^{-1}\right)+b_{m}\right\}+\sum_{n=1}^{N}\left\{c_{n} \log _{2}\left(\left(1-e^{\rho_{n}^{\prime}}\right)\left(\bar{h}_{n, n}-\bar{\varepsilon}_{n}\right) p_{n} q_{n}^{-1}\right)+d_{n}\right\}-\widehat{f}(m, n) \\
& \text { s.t. } \breve{\mathrm{C}}_{1}: 0 \leqslant e^{\rho_{n}^{\prime}} \leqslant 1, \quad \forall n, \\
& \breve{\mathrm{C}}_{2}:\left(1-e^{\rho_{n}^{\prime}}\right) p_{n}\left(\bar{h}_{n, n}-\bar{\varepsilon}_{n}\right) \geqslant q_{n} 2^{\left(R_{n}^{\text {D,min }}-d_{n}\right) / c_{n}}, \quad \forall n, \\
& \breve{\mathrm{C}}_{6}: \varsigma e^{\rho_{n}^{\prime}}\left\{\sigma_{n}^{2}+p_{n}\left(\bar{h}_{n, n}-\bar{\varepsilon}_{n}\right)+\sum_{m=1}^{M} x_{n, m} P_{m}\left(\bar{g}_{m, n}-v_{n}\right)\right\} \geqslant E_{n}^{\min }, \quad \forall n, \\
& \breve{\mathrm{C}}_{12}: q_{n} \geqslant \sigma_{n, \mathrm{ID}}^{2}+\left(1-e^{\rho_{n}^{\prime}}\right)\left(\sigma_{n}^{2}+\sum_{m=1}^{M} x_{n, m} P_{m}\left(\bar{g}_{m, n}+v_{n}\right)\right), \quad \forall n,
\end{aligned}
$$

其中, $\widehat{f}(m, n)=\eta\left\{\sum_{m=1}^{M} P_{m}+\sum_{n=1}^{N} p_{n}-\sum_{n=1}^{N} \varsigma e^{\rho_{n}^{\prime}}\left(\sigma_{n}^{2}+p_{n}\left(\bar{h}_{n, n}-\bar{\varepsilon}_{n}\right)+\sum_{m=1}^{M} x_{n, m} P_{m}\left(\bar{g}_{m, n}-v_{n}\right)\right)\right\}$. 因 为对数 - 指数和函数为凸函数 ${ }^{[17]}$, 所以问题 (38) 为凸优化问题, 可以通过拉格朗日对偶理论得到最 优的 $\rho_{n}^{*}$. 构建如下拉格朗日函数:

$$
L\left(o_{n}\right)=\sum_{m=1}^{M}\left\{a_{m} \log _{2}\left(P_{m} g_{m, 0} z_{m}^{-1}\right)+b_{m}\right\}+\sum_{n=1}^{N}\left\{c_{n} \log _{2}\left(\left(1-e^{\rho_{n}^{\prime}}\right) p_{n}\left(\bar{h}_{n, n}-\bar{\varepsilon}_{n}\right) q_{n}^{-1}\right)+d_{n}\right\}
$$




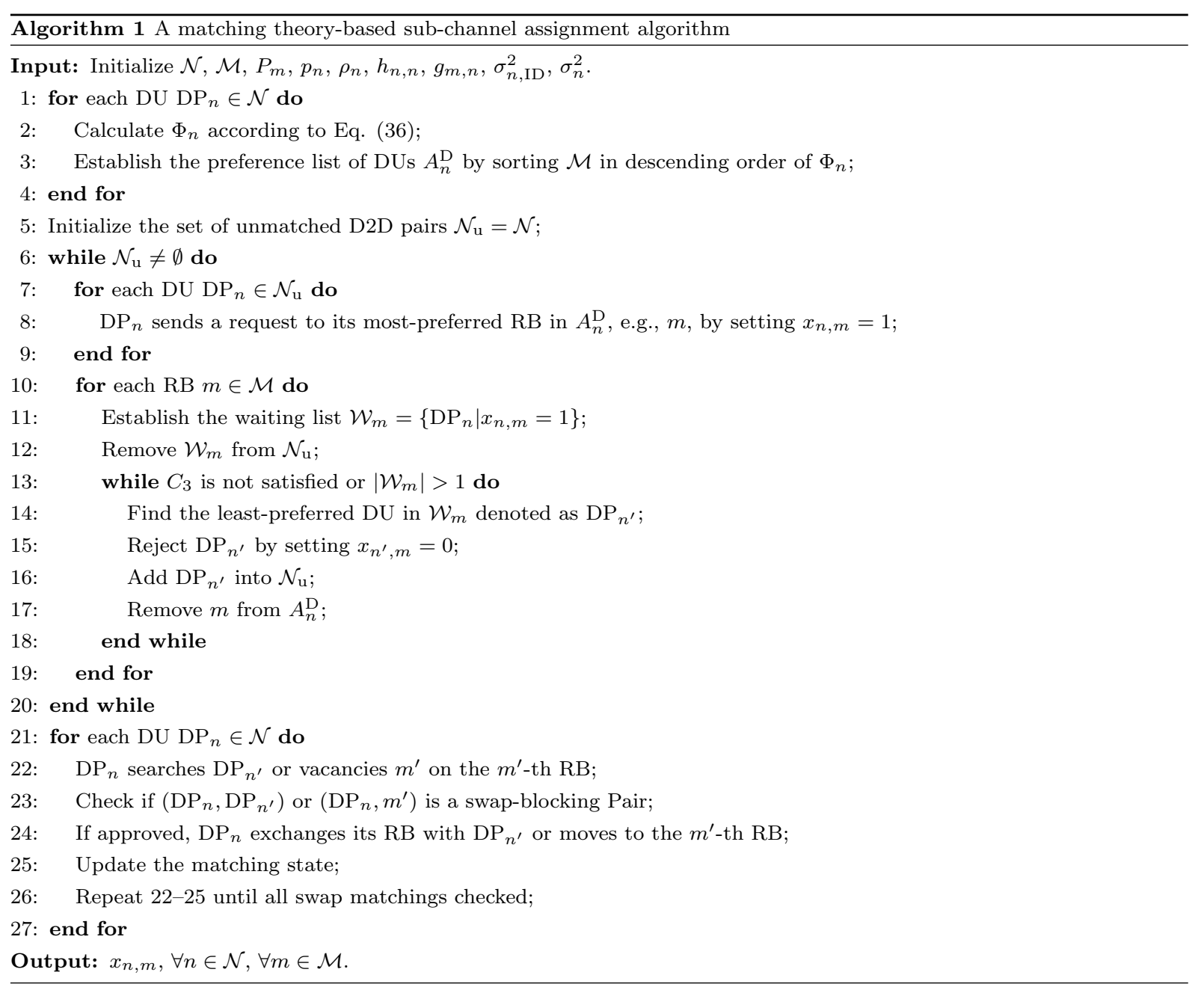

$$
\begin{aligned}
& -\eta\left\{\sum_{m=1}^{M} P_{m}+\sum_{n=1}^{N} p_{n}-\sum_{n=1}^{N} \varsigma e^{\rho_{n}^{\prime}}\left(\sigma_{n}^{2}+p_{n}\left(\bar{h}_{n, n}-\bar{\varepsilon}_{n}\right)+\sum_{m=1}^{M} x_{n, m} P_{m}\left(\bar{g}_{m, n}-v_{n}\right)\right)\right\} \\
& +\sum_{n=1}^{N} \nu_{n}\left(1-e^{\rho_{n}^{\prime}}\right)+\sum_{n=1}^{N} \tau_{n}\left(\left(1-e^{\rho_{n}^{\prime}}\right) p_{n}\left(\bar{h}_{n, n}-\bar{\varepsilon}_{n}\right)-q_{n} 2^{\left(R_{n}^{\mathrm{D}, \mathrm{min}}-d_{n}\right) / c_{n}}\right)-\eta\left(P_{\text {cir }}+N p_{\text {cir }}\right) \\
& +\sum_{n=1}^{N} \mu_{n}\left\{\varsigma e^{\rho_{n}^{\prime}}\left\{\sigma_{n}^{2}+p_{n}\left(\bar{h}_{n, n}-\bar{\varepsilon}_{n}\right)+\sum_{m=1}^{M} x_{n, m} P_{m}\left(\bar{g}_{m, n}-v_{n}\right)\right\}-E_{n}^{\min }\right\} \\
& +\sum_{n=1}^{N} \omega_{n}\left\{q_{n}-\left\{\sigma_{n, \mathrm{ID}}^{2}+\left(1-e^{\rho_{n}^{\prime}}\right)\left(\sigma_{n}^{2}+\sum_{m=1}^{M} x_{n, m} P_{m}\left(\bar{g}_{m, n}+v_{n}\right)\right)\right\}\right\},
\end{aligned}
$$

其中, $o_{n}=\left[\rho_{n}^{\prime}, \nu_{n}, \tau_{n}, \mu_{n}, \omega_{n}\right], \nu_{n}, \tau_{n}, \mu_{n}$ 和 $\omega_{n}$ 是非负的拉格朗日乘子. 对式 (39) 重新整理可得

$$
\begin{aligned}
L\left(o_{n}\right)= & \sum_{n=1}^{N} L_{n}\left(o_{n}\right)+\sum_{m=1}^{M}\left\{a_{m} \log _{2}\left(P_{m} g_{m, 0} z_{m}^{-1}\right)+b_{m}\right\}+\sum_{n=1}^{N} d_{n}-\sum_{n=1}^{N} \mu_{n} E_{n}^{\mathrm{min}}+\sum_{n=1}^{N} \nu_{n} \\
& -\eta\left\{\sum_{m=1}^{M} P_{m}+P_{\text {cir }}+\sum_{n=1}^{N} p_{n}+N p_{\text {cir }}\right\}+\sum_{n=1}^{N} \tau_{n}\left(p_{n}\left(\bar{h}_{n, n}-\bar{\varepsilon}_{n}\right)-q_{n} 2^{\left(R_{n}^{\mathrm{D}, \mathrm{min}}-d_{n}\right) / c_{n}}\right)
\end{aligned}
$$




$$
+\sum_{n=1}^{N} \omega_{n}\left\{q_{n}-\sigma_{n, \mathrm{ID}}^{2}-\left(\sigma_{n}^{2}+\sum_{m=1}^{M} x_{n, m} P_{m}\left(\bar{g}_{m, n}+v_{n}\right)\right)\right\}
$$

其中,

$$
\begin{aligned}
L_{n}\left(o_{n}\right)= & c_{n} \log _{2}\left(\left(1-e^{\rho_{n}^{\prime}}\right)\left(\bar{h}_{n, n}-\bar{\varepsilon}_{n}\right) p_{n} q_{n}^{-1}\right)-\sum_{n=1}^{N} \nu_{n} e^{\rho_{n}^{\prime}} \\
& +\left(\eta+\mu_{n}\right) \varsigma e^{\rho_{n}^{\prime}}\left(\sigma_{n}^{2}+p_{n}\left(\bar{h}_{n, n}-\bar{\varepsilon}_{n}\right)+\sum_{m=1}^{M} x_{n, m} P_{m}\left(\bar{g}_{m, n}-v_{n}\right)\right) \\
& -\sum_{n=1}^{N} \tau_{n} e^{\rho_{n}^{\prime}} p_{n}\left(\bar{h}_{n, n}-\bar{\varepsilon}_{n}\right)+\sum_{n=1}^{N} \omega_{n} e^{\rho_{n}^{\prime}}\left(\sigma_{n}^{2}+\sum_{m=1}^{M} x_{n, m} P_{m}\left(\bar{g}_{m, n}+v_{n}\right)\right)
\end{aligned}
$$

则, 问题 (38) 的对偶问题为

$$
\begin{aligned}
& \min _{\nu_{n}, \tau_{n}, \mu_{n}, \omega_{n}} D\left(\nu_{n}, \tau_{n}, \mu_{n}, \omega_{n}\right) \\
& \text { s.t. } \nu_{n} \geqslant 0, \tau_{n} \geqslant 0, \mu_{n} \geqslant 0, \omega_{n} \geqslant 0,
\end{aligned}
$$

其中, $D\left(\nu_{n}, \tau_{n}, \mu_{n}, \omega_{n}\right)=\max _{\rho_{n}^{\prime}, \eta} L_{n}\left(\rho_{n}^{\prime}, \eta\right)$.

根据卡罗需 - 库恩 - 塔克 (Karush-Kuhn-Tucker) 条件 ${ }^{[17]}$, 令 $\frac{\partial L_{n}\left(o_{n}\right)}{\partial e^{\rho_{n}^{\prime}}}=0$, 可以得到 $\rho_{n}^{*}$ 的解析 解为

$$
\rho_{n}^{*}=e^{\rho_{n}^{\prime * *}}=\left[1+\frac{c_{n}}{F_{n} \ln 2}\right]^{+}
$$

其中, $[x]^{+}=\max (0, x)$,

$$
\begin{aligned}
F_{n}= & \left(\eta+\mu_{n}\right) \varsigma\left(\sigma_{n}^{2}+p_{n}\left(\bar{h}_{n, n}-\bar{\varepsilon}_{n}\right)+\sum_{m=1}^{M} x_{n, m} P_{m}\left(\bar{g}_{m, n}-v_{n}\right)\right) \\
& +\omega_{n}\left(\sigma_{n}^{2}+\sum_{m=1}^{M} x_{n, m} P_{m}\left(\bar{g}_{m, n}+v_{n}\right)\right)-\nu_{n}-\tau_{n} p_{n}\left(\bar{h}_{n, n}-\bar{\varepsilon}_{n}\right) .
\end{aligned}
$$

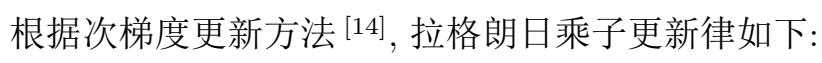

$$
\begin{aligned}
& \nu_{n}^{t+1}=\left[\nu_{n}^{t}-d_{1}^{t} \times\left(1-e^{\rho_{n}^{\prime}}\right)\right]^{+}, \\
& \tau_{n}^{t+1}=\left[\tau_{n}^{t}-d_{2}^{t} \times\left(\left(1-e^{\rho_{n}^{\prime}}\right) p_{n}\left(\bar{h}_{n, n}-\bar{\varepsilon}_{n}\right)-q_{n} 2^{\left(R_{n}^{\mathrm{D}, \min }-d_{n}\right) / c_{n}}\right)\right]^{+}, \\
& \omega_{n}^{t+1}=\left[\omega_{n}^{t}-d_{3}^{t} \times\left\{q_{n}-\sigma_{n, \mathrm{ID}}^{2}-\left(\sigma_{n}^{2}+\sum_{m=1}^{M} x_{n, m} P_{m}\left(\bar{g}_{m, n}+v_{n}\right)\right)\right\}\right]^{+}, \\
& \mu_{n}^{t+1}=\left[\mu_{n}^{t}-d_{4}^{t} \times\left\{\varsigma e^{\rho_{n}^{\prime}}\left\{\sigma_{n}^{2}+p_{n}\left(\bar{h}_{n, n}-\bar{\varepsilon}_{n}\right)+\sum_{m=1}^{M} x_{n, m} P_{m}\left(\bar{g}_{m, n}-v_{n}\right)\right\}-E_{n}^{\min }\right\}\right]^{+},
\end{aligned}
$$

其中, $t$ 表示迭代次数, $d_{1}-d_{4}$ 为迭代步长. 通过选择合适的步长, 可以保证上述迭代算法收玫 ${ }^{[14]}$. 


\section{2 功率分配优化子问题求解}

定义 $p_{n}=e^{p_{n}^{\prime}}, P_{m}=e^{P_{m}^{\prime}}, q_{n}=e^{q_{n}^{\prime}}$ 和 $z_{m}=e^{z_{m}^{\prime}}$, 在确定的 $\rho_{n}^{*}$ 条件下, 问题 (35) 可以转化为如下 功率分配子问题:

$$
\begin{array}{ll} 
& \max _{P_{m}^{\prime}, p_{n}^{\prime}, \eta, q_{n}^{\prime}, z_{m}^{\prime}} \tilde{f}(\eta) \\
\text { s.t. } & \hat{\mathrm{C}}_{2}:\left(1-\rho_{n}\right) e^{p_{n}^{\prime}}\left(\bar{h}_{n, n}-\bar{\varepsilon}_{n}\right) \geqslant e^{q_{n}^{\prime}} 2^{\left(R_{n}^{\mathrm{D}, \min }-d_{n}\right) / c_{n}}, \forall n, \\
& \hat{\mathrm{C}}_{3}: e^{P_{m}^{\prime}} g_{m, 0} \geqslant e^{z_{m}^{\prime}} 2^{\left(R_{m}^{\mathrm{C}, \min }-b_{m}\right) / a_{m}}, \forall m, \\
& \hat{\mathrm{C}}_{4}: 0 \leqslant e^{p_{n}^{\prime}} \leqslant p_{n}^{\max }, \forall n, \\
& \hat{\mathrm{C}}_{5}: 0 \leqslant e^{P_{m}^{\prime}} \leqslant P_{m}^{\max }, \forall m, \\
& \hat{\mathrm{C}}_{6}: \varsigma \rho_{n}\left\{\sigma_{n}^{2}+e^{p_{n}^{\prime}}\left(\bar{h}_{n, n}-\bar{\varepsilon}_{n}\right)+\sum_{m=1}^{M} x_{n, m} e^{P_{m}^{\prime}}\left(\bar{g}_{m, n}-v_{n}\right)\right\} \geqslant E_{n}^{\min }, \forall n, \\
& \hat{\mathrm{C}}_{11}: e^{z_{m}^{\prime}} \geqslant \sigma^{2}+\sum_{n=1}^{N} x_{n, m} e^{p_{n}^{\prime}}\left(\bar{h}_{n, 0}+\xi_{0}\right), \forall m, \\
& \hat{\mathrm{C}}_{12}: e^{q_{n}^{\prime}} \geqslant \sigma_{n, \mathrm{ID}}^{2}+\left(1-\rho_{n}\right)\left(\sigma_{n}^{2}+\sum_{m=1}^{M} x_{n, m} e^{P_{m}^{\prime}}\left(\bar{g}_{m, n}+v_{n}\right)\right), \forall n,
\end{array}
$$

其中,

$$
\begin{aligned}
\tilde{f}(\eta)= & \sum_{m=1}^{M}\left\{a_{m} \log _{2}\left(e^{P_{m}^{\prime}-z_{m}^{\prime}} g_{m, 0}\right)+b_{m}\right\}+\sum_{n=1}^{N}\left\{c_{n} \log _{2}\left(\left(1-\rho_{n}\right) e^{p_{n}^{\prime}-q_{n}^{\prime}}\left(\bar{h}_{n, n}-\bar{\varepsilon}_{n}\right)\right)+d_{n}\right\}-\eta P_{\text {cir }} \\
& -\eta\left\{\sum_{m=1}^{M} e^{P_{m}^{\prime}}+\sum_{n=1}^{N} e^{p_{n}^{\prime}}+N p_{\text {cir }}-\sum_{n=1}^{N} \varsigma \rho_{n}\left(\sigma_{n}^{2}+e^{p_{n}^{\prime}}\left(\bar{h}_{n, n}-\bar{\varepsilon}_{n}\right)+\sum_{m=1}^{M} x_{n, m} e^{P_{m}^{\prime}}\left(\bar{g}_{m, n}-v_{n}\right)\right)\right\} .
\end{aligned}
$$

因为对数 - 指数和函数为凸函数, 所以问题 (49) 是一个凸优化问题. 因此构建如下拉格朗日函数:

$$
\begin{aligned}
L\left(\Theta_{n, m}\right)= & \sum_{m=1}^{M}\left\{a_{m} \log _{2}\left(e^{P_{m}^{\prime}-z_{m}^{\prime}} g_{m, 0}\right)+b_{m}\right\}+\sum_{n=1}^{N}\left\{c_{n} \log _{2}\left(\left(1-\rho_{n}\right) e^{p_{n}^{\prime}-q_{n}^{\prime}}\left(\bar{h}_{n, n}-\bar{\varepsilon}_{n}\right)\right)+d_{n}\right\} \\
& +\sum_{n=1}^{N} \beta_{n}\left\{p_{n}^{\max }-e^{p_{n}^{\prime}}\right\}+\sum_{m=1}^{M} \alpha_{m}\left\{P_{m}^{\max }-e^{P_{m}^{\prime}}\right\}-\eta\left\{\sum_{m=1}^{M} e^{P_{m}^{\prime}}+P_{\text {cir }}+\sum_{n=1}^{N} e^{p_{n}^{\prime}}+N p_{\text {cir }}\right\} \\
& +\sum_{m=1}^{M} \phi_{m}\left\{e^{z_{m}^{\prime}}-\sigma^{2}-\sum_{n=1}^{N} x_{n, m} e^{p_{n}^{\prime}}\left(\bar{h}_{n, 0}+\xi_{0}\right)\right\}+\sum_{m=1}^{M} \chi_{m}\left\{e^{P_{m}^{\prime}} g_{m, 0}-e^{z_{m}^{\prime}} 2^{\left(R_{m}^{\mathrm{C}, \min }-b_{m}\right) / a_{m}}\right\} \\
& +\sum_{n=1}^{N} \theta_{n}\left\{\varsigma \rho_{n}\left(\sigma_{n}^{2}+e^{p_{n}^{\prime}}\left(\bar{h}_{n, n}-\bar{\varepsilon}_{n}\right)+\sum_{m=1}^{M} x_{n, m} e^{P_{m}^{\prime}}\left(\bar{g}_{m, n}-v_{n}\right)\right)-E_{n}^{\min }\right\} \\
& +\sum_{n=1}^{N} \varphi_{n}\left\{e^{q_{n}^{\prime}}-\sigma_{n, \mathrm{ID}}^{2}-\left(1-\rho_{n}\right)\left(\sigma_{n}^{2}+\sum_{m=1}^{M} x_{n, m} e^{P_{m}^{\prime}}\left(\bar{g}_{m, n}+v_{n}\right)\right)\right\} \\
& +\eta \sum_{n=1}^{N} \varsigma \rho_{n}\left(\sigma_{n}^{2}+e^{p_{n}^{\prime}}\left(\bar{h}_{n, n}-\bar{\varepsilon}_{n}\right)+\sum_{m=1}^{M} x_{n, m} e^{P_{m}^{\prime}}\left(\bar{g}_{m, n}-v_{n}\right)\right) \\
& +\sum_{n=1}^{N} \lambda_{n}\left\{\left(1-\rho_{n}\right) e^{p_{n}^{\prime}}\left(\bar{h}_{n, n}-\bar{\varepsilon}_{n}\right)-e^{q_{n}^{\prime}} 2^{\left(R_{n}^{\mathrm{D}, \min }-d_{n}\right) / c_{n}}\right\},
\end{aligned}
$$


其中, $\Theta_{n, m}=\left[P_{m}^{\prime}, p_{n}^{\prime}, z_{m}^{\prime}, q_{n}^{\prime}, \eta, \lambda_{n}, \chi_{m}, \beta_{n}, \alpha_{m}, \theta_{n}, \phi_{m}, \varphi_{n}\right], \lambda_{n}, \chi_{m}, \beta_{n}, \alpha_{m}, \theta_{n}, \phi_{m}$ 和 $\varphi_{n}$ 为非负的拉 格朗日乘子, 式 (51) 可以重新描述为

$$
\begin{aligned}
L\left(\Theta_{n, m}\right)= & \sum_{m=1}^{M} \sum_{n=1}^{N} L_{n, m}\left(\Theta_{n, m}\right)+\sum_{m=1}^{M} b_{m}+\sum_{n=1}^{N} d_{n}-\eta P_{\text {cir }}+\eta \sum_{n=1}^{N} \varsigma \rho_{n} \sigma_{n}^{2}+\sum_{n=1}^{N} \beta_{n} p_{n}^{\max }-\eta N p_{\text {cir }} \\
& +\sum_{m=1}^{M} \alpha_{m} P_{m}^{\max }+\sum_{n=1}^{N} \theta_{n}\left(\varsigma \rho_{n} \sigma_{n}^{2}-E_{n}^{\min }\right)-\sum_{m=1}^{M} \phi_{m} \sigma^{2}+\sum_{n=1}^{N} \varphi_{n}\left\{-\sigma_{n, \mathrm{ID}}^{2}-\left(1-\rho_{n}\right) \sigma_{n}^{2}\right\}
\end{aligned}
$$

其中,

$$
\begin{aligned}
& L_{n, m}\left(\Theta_{n, m}\right) \\
& =a_{m} \log _{2}\left(e^{P_{m}^{\prime}-z_{m}^{\prime}} g_{m, 0}\right) / N+c_{n} \log _{2}\left(\left(1-\rho_{n}\right) e^{p_{n}^{\prime}-q_{n}^{\prime}}\left(\bar{h}_{n, n}-\bar{\varepsilon}_{n}\right)\right) / M \\
& +e^{p_{n}^{\prime}}\left\{\left(\eta+\theta_{n}\right) \varsigma \rho_{n}\left(\bar{h}_{n, n}-\bar{\varepsilon}_{n}\right)+\lambda_{n}\left(1-\rho_{n}\right)\left(\bar{h}_{n, n}-\bar{\varepsilon}_{n}\right)-\eta-\beta_{n}-\sum_{m=1}^{M} \phi_{m} x_{n, m}\left(\bar{h}_{n, 0}+\xi_{0}\right)\right\} / M \\
& +e^{P_{m}^{\prime}}\left\{\chi_{m} g_{m, 0}-\alpha_{m}-\eta+\sum_{n=1}^{N}\left(\left(\theta_{n}+\eta\right) \varsigma \rho_{n} x_{n, m}\left(\bar{g}_{m, n}-v_{n}\right)-\varphi_{n}\left(1-\rho_{n}\right) x_{n, m}\left(\bar{g}_{m, n}+v_{n}\right)\right)\right\} / N \\
& -e^{q_{n}^{\prime}}\left\{\lambda_{n} 2^{\left(R_{n}^{\mathrm{D}, \min }-d_{n}\right) / c_{n}}-\varphi_{n}\right\} / M-e^{z_{m}^{\prime}}\left\{\chi_{m} 2^{\left(R_{m}^{\mathrm{C}, \min }-b_{m}\right) / a_{m}}-\phi_{m}\right\} / N .
\end{aligned}
$$

因此, 问题 (49) 的对偶问题为

$$
\begin{aligned}
& \min _{\lambda_{n}, \chi_{m}, \beta_{n}, \alpha_{m}, \theta_{n}, \phi_{m}, \varphi_{n}} D\left(\lambda_{n}, \chi_{m}, \beta_{n}, \alpha_{m}, \theta_{n}, \phi_{m}, \varphi_{n}\right) \\
& \text { s.t. } \lambda_{n} \geqslant 0, \chi_{m} \geqslant 0, \beta_{n} \geqslant 0, \alpha_{m} \geqslant 0, \theta_{n} \geqslant 0, \phi_{m} \geqslant 0, \varphi_{n} \geqslant 0,
\end{aligned}
$$

其中,

$$
D\left(\lambda_{n}, \chi_{m}, \beta_{n}, \alpha_{m}, \theta_{n}, \phi_{m}, \varphi_{n}\right)=\max _{P_{m}^{\prime}, p_{n}^{\prime}, z_{m}^{\prime}, q_{n}^{\prime}, \eta} L_{n, m}\left(P_{m}^{\prime}, p_{n}^{\prime}, z_{m}^{\prime}, q_{n}^{\prime}, \eta\right) .
$$

同理, 根据卡罗需 - 库恩 - 塔克条件, 可以得到最优功率的解析解为

$$
\begin{aligned}
& p_{n}^{*}=e^{p_{n}^{\prime *}}=\left[\frac{c_{n}}{B_{n} \ln 2}\right]^{+}, \\
& P_{m}^{*}=e^{P_{m}^{\prime \prime *}}=\left[\frac{a_{m}}{A_{m} \ln 2}\right]^{+}, \\
& q_{n}^{*}=e^{q_{n}^{\prime, *}}=\left[c_{n} /\left(\left(\varphi_{n}-\lambda_{n} 2^{\left(R_{n}^{\mathrm{D}, \mathrm{min}}-d_{n}\right) / c_{n}}\right) \ln 2\right)\right]^{+}, \\
& z_{m}^{*}=e^{z_{m}^{\prime \prime *}}=\left[a_{m} /\left(\left(\phi_{m}-\chi_{m} 2^{\left(R_{m}^{\mathrm{C}, \min }-b_{m}\right) / a_{m}}\right) \ln 2\right)\right]^{+},
\end{aligned}
$$

其中,

$$
\begin{aligned}
& B_{n}=-\left(\eta+\theta_{n}\right) \varsigma \rho_{n}\left(\bar{h}_{n, n}-\bar{\varepsilon}_{n}\right)+\eta+\beta_{n}-\lambda_{n}\left(1-\rho_{n}\right)\left(\bar{h}_{n, n}-\bar{\varepsilon}_{n}\right)+\sum_{m=1}^{M} \phi_{m} x_{n, m}\left(\bar{h}_{n, 0}+\xi_{0}\right), \\
& A_{m}=-\sum_{n=1}^{N}\left(\left(\theta_{n}+\eta\right) \varsigma \rho_{n} x_{n, m}\left(\bar{g}_{m, n}-v_{n}\right)-\varphi_{n}\left(1-\rho_{n}\right) x_{n, m}\left(\bar{g}_{m, n}+v_{n}\right)\right)-\chi_{m} g_{m, 0}+\alpha_{m}+\eta
\end{aligned}
$$


根据次梯度更新方法, 拉格朗日乘子更新律如下:

$$
\begin{aligned}
\beta_{n}^{t+1} & =\left[\beta_{n}^{t}-d_{5}^{t} \times\left(p_{n}^{\max }-e^{p_{n}^{\prime}}\right)\right]^{+}, \\
\alpha_{m}^{t+1} & =\left[\alpha_{m}^{t}-d_{6}^{t} \times\left(P_{m}^{\max }-e^{P_{m}^{\prime}}\right)\right]^{+}, \\
\chi_{m}^{t+1} & =\left[\chi_{m}^{t}-d_{7}^{t} \times\left(e^{P_{m}^{\prime}} g_{m, 0}-e^{z_{m}^{\prime}} 2^{\left(R_{m}^{\mathrm{C}, \mathrm{min}}-b_{m}\right) / a_{m}}\right)\right]^{+}, \\
\phi_{m}^{t+1} & =\left[\phi_{m}^{t}-d_{8}^{t} \times\left(e^{z_{m}^{\prime}}-\sigma^{2}-\sum_{n=1}^{N} x_{n, m} e^{p_{n}^{\prime}}\left(\bar{h}_{n, 0}+\xi_{0}\right)\right)\right]^{+}, \\
\lambda_{n}^{t+1}= & {\left[\lambda_{n}^{t}-d_{9}^{t} \times\left(\left(1-\rho_{n}\right) e^{p_{n}^{\prime}}\left(\bar{h}_{n, n}-\bar{\varepsilon}_{n}\right)-e^{q_{n}^{\prime}} 2^{\left(R_{n}^{\mathrm{D}, \mathrm{min}}-d_{n}\right) / c_{n}}\right)\right]^{+}, } \\
\varphi_{n}^{t+1} & =\left[\varphi_{n}^{t}-d_{10}^{t} \times\left(e^{q_{n}^{\prime}}-\sigma_{n, \mathrm{ID}}^{2}-\left(1-\rho_{n}\right)\left(\sigma_{n}^{2}+\sum_{m=1}^{M} x_{n, m} e^{P_{m}^{\prime}}\left(\bar{g}_{m, n}+v_{n}\right)\right)\right)\right]^{+}, \\
\theta_{n}^{t+1} & =\left[\theta_{n}^{t}-d_{11}^{t} \times\left(\varsigma \rho_{n}\left(\sigma_{n}^{2}+e^{p_{n}^{\prime}}\left(\bar{h}_{n, n}-\bar{\varepsilon}_{n}\right)+\sum_{m=1}^{M} x_{n, m} e^{P_{m}^{\prime}}\left(\bar{g}_{m, n}-v_{n}\right)\right)-E_{n}^{\min }\right)\right]^{+},
\end{aligned}
$$

其中, $d_{5}-d_{11}$ 为迭代步长, 所提资源分配算法的步骤如算法 2 所示.

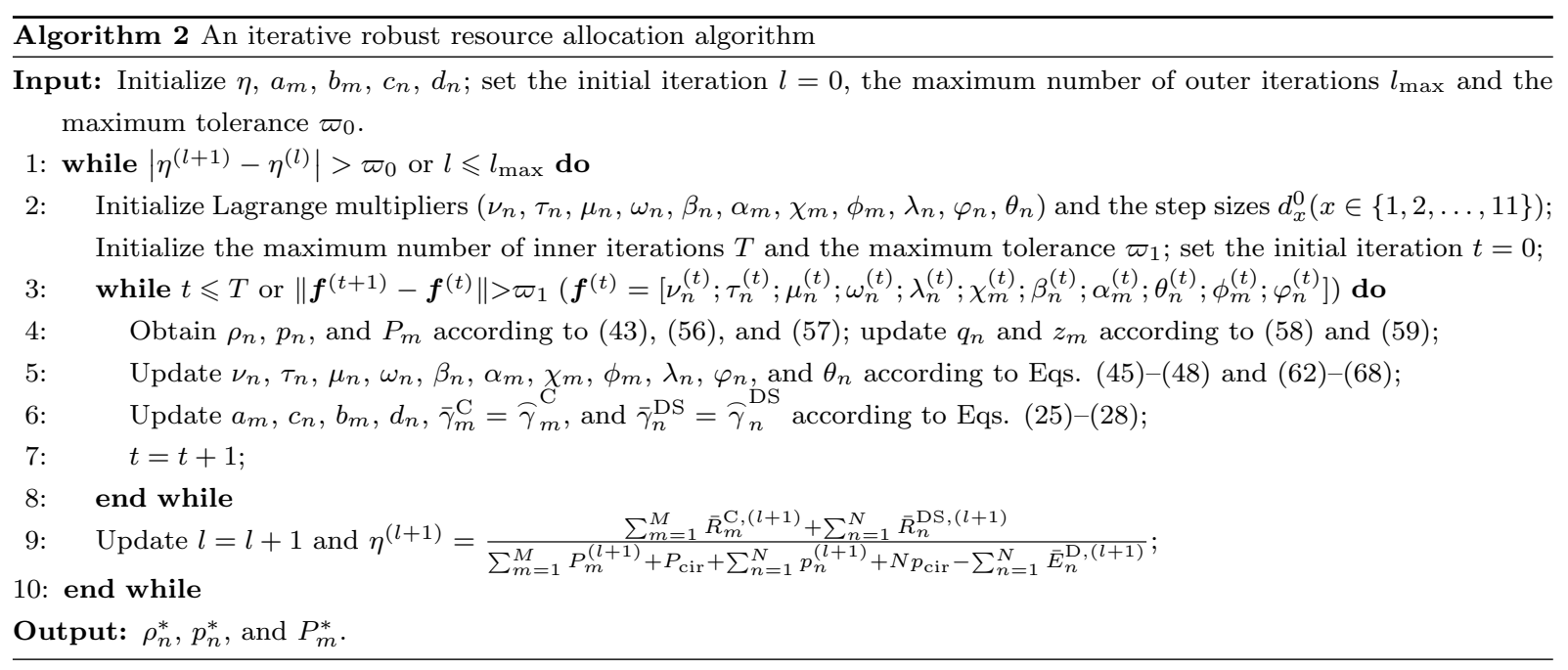

\section{5 仿真结果}

为了验证所提算法 (proposed algorithm) 的有效性, 将其与现有算法进行对比: 非鲁棒资源分配算 法 ${ }^{[9]}$ (non-robust algorithm)、最大速率鲁棒算法 ${ }^{[13]}$ (max-rate algorithm) 和随机匹配鲁棒资源分配算 法 $^{2)}$ (random matching algorithm). 假设网络中系统参数如下: $M=2, N=2, \varsigma=0.8, \sigma^{2}=10^{-5} \mathrm{~W}$, $\sigma_{n}^{2}=10^{-5} \mathrm{~W}, \sigma_{n, \mathrm{ID}}^{2}=10^{-5} \mathrm{~W}, l_{\max }=10^{4}, T=10^{4}, \varpi_{0}=10^{-5}, \varpi_{1}=10^{-5}, P_{\text {cir }}=0.04 \mathrm{~W}, p_{\text {cir }}$

2) 本文算法考虑了信道不确定性对系统能效的影响, 为了验证算法的鲁棒性, 因此需要与没有考虑信道不确定性 的能效最大化算法进行对比, 即, 非鲁棒资源分配算法. 为了说明本文算法具有较好的能效性能, 因此与考虑信道不确 定性的和速率最大化算法进行对比, 即, 最大速率鲁棒算法. 进一步, 为了体现本文匹配算法信道资源分配的有效性, 因 此与随机匹配算法对比来验证本文算法性能. 

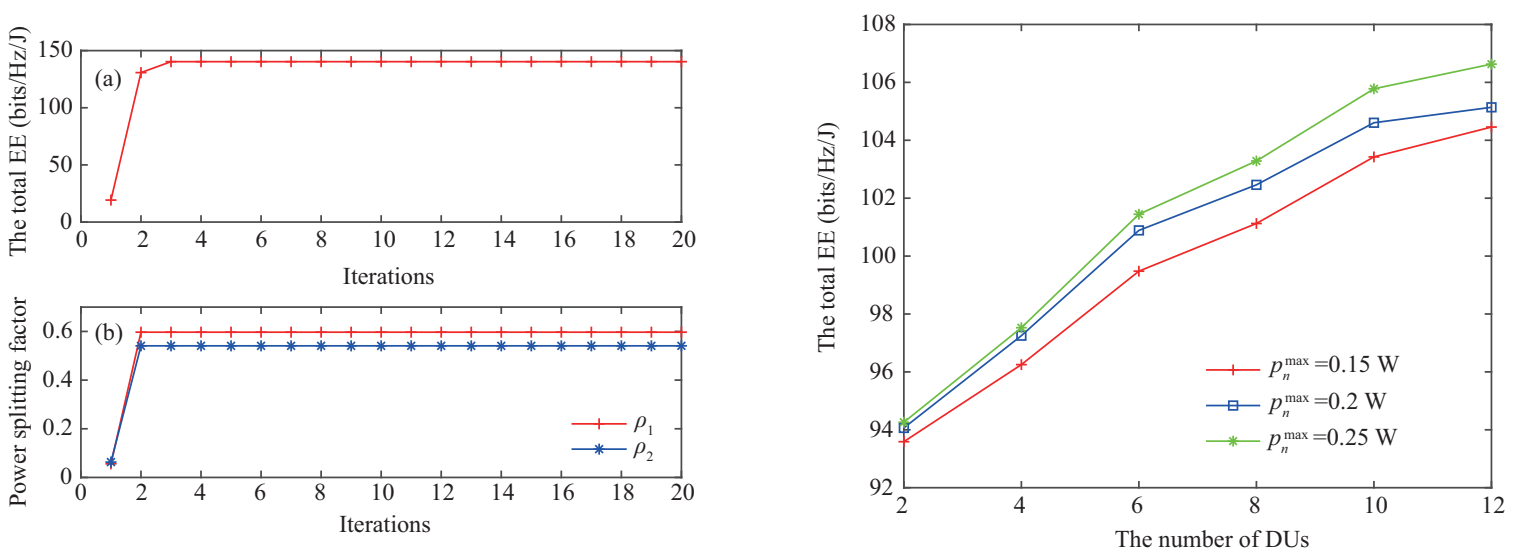

图 2 (网络版彩图) 系统总能效与功率分流因子收敛性 能 $\left(\varepsilon_{n}^{2}=0.01, v_{n}^{2}=0.01, \xi_{0}^{2}=0.01\right)$

Figure 2 (Color online) The convergence of the total EE and PS factor $\left(\varepsilon_{n}^{2}=0.01, v_{n}^{2}=0.01, \xi_{0}^{2}=0.01\right)$. (a) The total EE versus iterations; (b) power splitting factor versus 图 3 (网络版彩图) 系统总能效与 D2D 用户对数量的 关系 $\left(\varepsilon_{n}^{2}=0.001, v_{n}^{2}=0.001, \xi_{0}^{2}=0.001\right)$

Figure 3 (Color online) The total EE versus the number of DUs $\left(\varepsilon_{n}^{2}=0.001, v_{n}^{2}=0.001, \xi_{0}^{2}=0.001\right)$ iterations
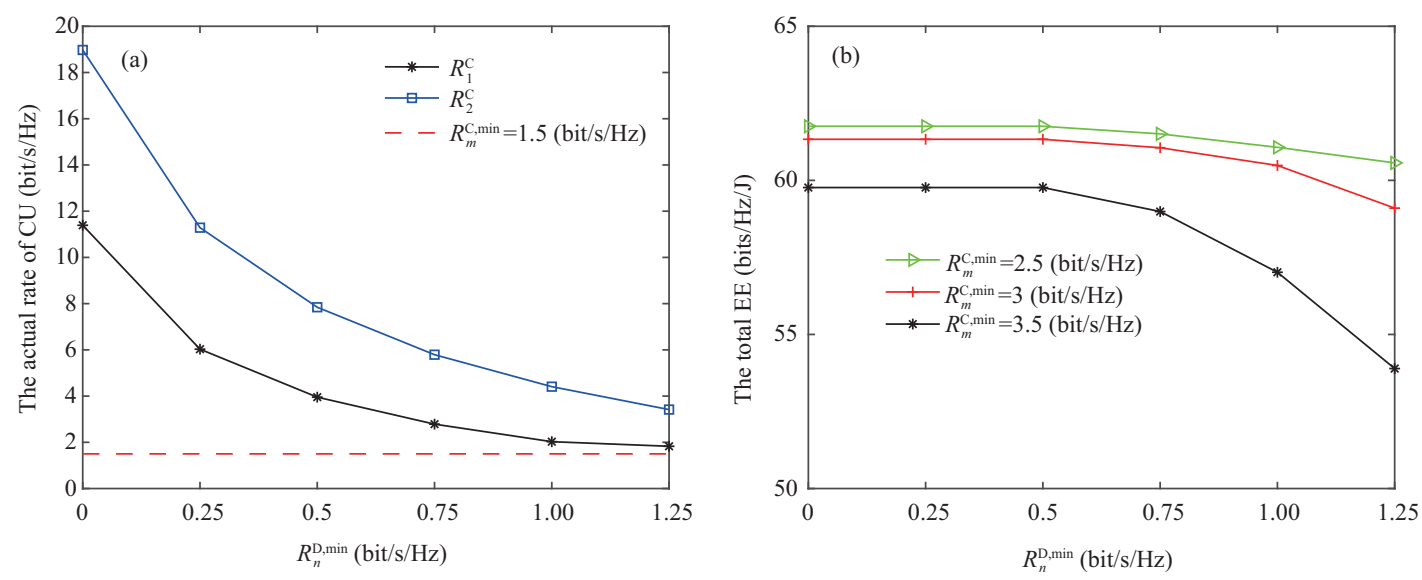

图 4 (网络版彩图) 蜂窝用户实际速率 $(\mathbf{a})$, 系统总能效 $(\mathbf{b})$ 与 $R_{n}^{\mathrm{D}, \mathrm{min}}$ 的关系 $\left(\varepsilon_{n}^{2}=0.01, v_{n}^{2}=0.01, \xi_{0}^{2}=0.01\right)$ Figure 4 (Color online) The actual rate of cellular user (a), the total EE (b) versus $R_{n}^{\mathrm{D}, \min }\left(\varepsilon_{n}^{2}=0.01, v_{n}^{2}=0.01\right.$, $\left.\xi_{0}^{2}=0.01\right)$

$=0.02 \mathrm{~W}, p_{n}^{\max }=0.25 \mathrm{~W}, P_{m}^{\max }=0.25 \mathrm{~W}, E_{n}^{\min }=0.004 \mathrm{~W}, R_{m}^{\mathrm{C}, \min }=2 \mathrm{bit} / \mathrm{s} / \mathrm{Hz}, R_{n}^{\mathrm{D}, \min }=1 \mathrm{bit} / \mathrm{s} / \mathrm{Hz}$, $\varepsilon_{n} \in[0,1], v_{n} \in[0,1], \xi_{0} \in[0,1]^{[14]}$.

图 2 给出了系统能效和功率分流因子的收玫性能. 从图中可以看出, 系统能效和功率分流因子能 够快速收敛. 说明所提出算法具有良好的收敛性.

图 3 给出了系统总能效与 $\mathrm{D} 2 \mathrm{D}$ 用户对数量之间的关系. 随着 $\mathrm{D} 2 \mathrm{D}$ 用户对数量的增加, 系统总能 效随之增加. 因为 $\mathrm{D} 2 \mathrm{D}$ 用户数量的增加会提升系统总的传输速率, 且传输速率的增加远远大于能耗 的增加, 从而使得系统总能效增加. 另一方面, 在相同 $\mathrm{D} 2 \mathrm{D}$ 用户对数量的情况下, 随着 $p_{n}^{\max }$ 的增大, 系统能效随之增大. 因为 $p_{n}^{\max }$ 的增大会增大传输功率的可行域, 从而使得能效增大.

图 4(a) 给出了蜂窝用户实际速率与 $R_{n}^{\mathrm{D}, \min }$ 的关系. 随着 $R_{n}^{\mathrm{D}, \min }$ 的增大, 蜂窝用户的实际速率减 

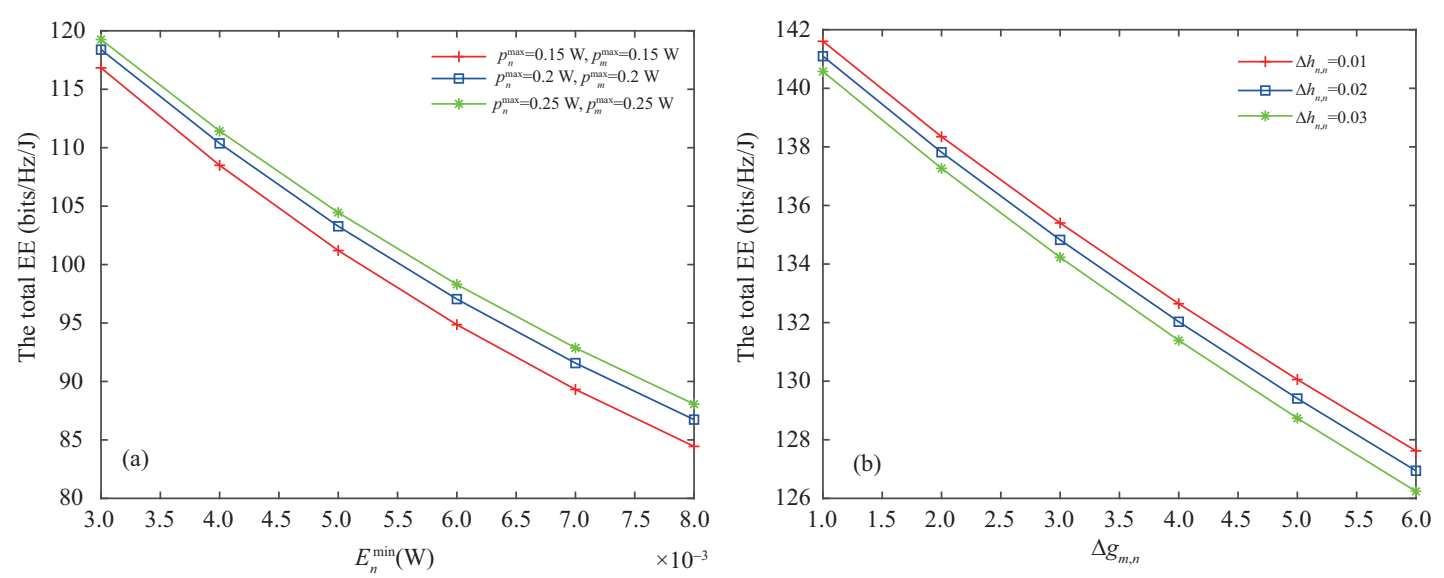

图 5 (网络版彩图) (a) 系统总能效与 $E_{n}^{\min }$ 之间的关系 $\left(\varepsilon_{n}^{2}=0.01, v_{n}^{2}=0.01, \xi_{0}^{2}=0.01\right) ;(\mathbf{b})$ 系统总能效与 $\Delta g_{m, n}$ 的关系 $\left(\xi_{0}^{2}=0.01\right)$

Figure 5 (Color online) The total EE versus $E_{n}^{\min }\left(\varepsilon_{n}^{2}=0.01, v_{n}^{2}=0.01, \xi_{0}^{2}=0.01\right)$; (b) the total EE versus $\Delta g_{m, n}$ $\left(\xi_{0}^{2}=0.01\right)$

小, 但仍然大于速率门限 $R_{m}^{\mathrm{C}, \mathrm{min}}=1.5 \mathrm{bit} / \mathrm{s} / \mathrm{Hz}$. 因为 $R_{n}^{\mathrm{D}, \mathrm{min}}$ 的增大, 会使得 $\mathrm{D} 2 \mathrm{D}$ 用户传输更大的功 率来满足自身最小速率需求, 且从 $C_{3}$ 可以看出会给蜂窝用户带来更多的干扰, 从而导致蜂窝用户的 实际速率是逐渐降低的.

图 4(b) 给出了系统总能效与 $R_{n}^{\mathrm{D}, \mathrm{min}}$ 的关系. 随着 $R_{n}^{\mathrm{D}, \min }$ 的增大, 系统总能效降低. 因为 $\mathrm{D} 2 \mathrm{D}$ 用户传输速率的增加低于功率消耗, 导致系统总能效降低. 在相同的 $R_{n}^{\mathrm{D}, \min }$ 情况下, 随着 $R_{m}^{\mathrm{C}, \mathrm{min}}$ 的 增大, 系统总能效降低. 原因是为了保证较大的 $R_{m}^{\mathrm{C}, \mathrm{min}}$, 蜂窝用户需要分配更多的功率, 从而导致能耗 增加.

图 5(a) 给出了系统总能效与 $E_{n}^{\min }$ 之间的关系. 随着 $E_{n}^{\min }$ 的增大, 系统总能效降低. 因为 $E_{n}^{\min }$ 增大, 从 $\mathrm{C}_{6}$ 可以看出会使得 $\mathrm{D} 2 \mathrm{D}$ 用户要求收集的能量也随之增加, 从而导致 D2D 用户的功耗增大、 系统能效降低. 在相同的 $E_{n}^{\min }$ 情况下, 随着 $p_{n}^{\max }$ 的增大, 系统的能效增加, 因为 $p_{n}^{\max }$ 的增大会使得 传输功率可行域增大, 从而增大传输速率.

图 5(b) 给出了系统总能效与 $\Delta g_{m, n}$ 的关系. 随着 $\Delta g_{m, n}$ 和 $\Delta h_{n, n}$ 的增大, 系统总能效降低. 结 合问题 (12) 和 (35) 可以看出, 为了克服信道不确定性的影响, 用户需要增加传输功率, 导致用户能耗 增加、能效降低.

图 6(a) 给出了不同算法下 D2D 用户实际速率与 $\Delta h_{n, n}$ 的关系. 随着 $\Delta h_{n, n}$ 的增大, D2D 用户 实际速率降低. 结合问题 (12) 和 (35) 可以看出, $\Delta h_{n, n}$ 的增大使得 D2D 用户需要更多的传输功率用 于克服信道不确定性, 从而引起 D2D 用户实际速率降低. 另外本文算法 $\mathrm{D} 2 \mathrm{D}$ 用户实际速率始终高于 $R_{n}^{\mathrm{D}, \min }$, 而非鲁棒算法在不确定性较大时 $\mathrm{D} 2 \mathrm{D}$ 用户实际速率低于 $R_{n}^{\mathrm{D}, \mathrm{min}}$, 从而导致通信中断.

图 6(b) 给出了不同算法下系统总能效与 $E_{n}^{\min }$ 的关系. 随着 $E_{n}^{\min }$ 的增加, 系统总能效降低, 本文 算法优于其他 3 种算法. 因为 $E_{n}^{\min }$ 的增加, 从 $\mathrm{C}_{6}$ 可以看出 $\mathrm{D} 2 \mathrm{D}$ 用户要求收集的能量也随之增加, 从而导致 D2D 用户的功耗增大、系统能效降低. 另外, 由于最大化速率算法的目标函数是 D2D 用户 的和速率, 没有考虑能效, 导致功率一直处于最大值, 接收机处收集的能量一直能够满足 $E_{n}^{\min }$ 要求, 所以当横轴 $E_{n}^{\min }$ 增加时, 不会影响最大化速率算法的功率选择策略, 导致能效保持不变. 

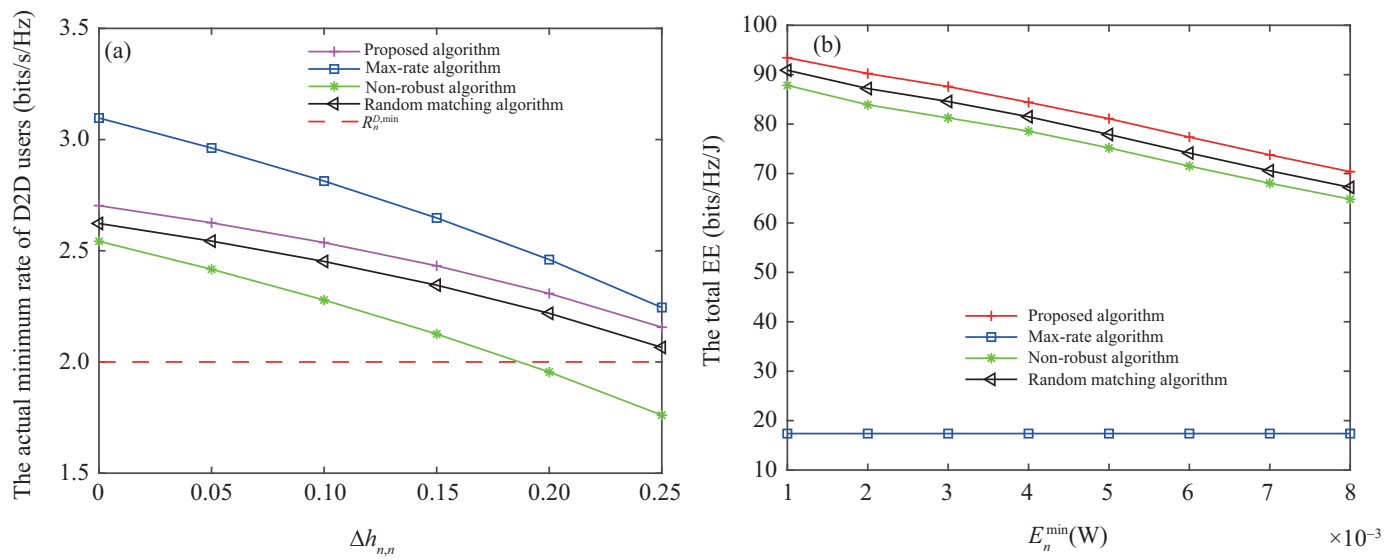

图 6 (网络版彩图) (a) 不同算法下 D2D 用户实际速率与 $\Delta h_{n, n}$ 的关系 $\left(p_{n}^{\max }=0.1 \mathrm{~W}, P_{m}^{\max }=0.1 \mathrm{~W}, v_{n}^{2}=0\right.$, $\left.\xi_{0}^{2}=0\right) ;(\mathbf{b})$ 不同算法下系统总能效与 $E_{n}^{\min }$ 的关系 $\left(\varepsilon_{n}^{2}=0.01, v_{n}^{2}=0.01, \xi_{0}^{2}=0.01\right)$

Figure 6 (Color online) (a) The actual rate of D2D user versus $\Delta h_{n, n}$ under different algorithms $\left(p_{n}^{\max }=0.1 \mathrm{~W}, P_{m}^{\max }=\right.$ $\left.0.1 \mathrm{~W}, v_{n}^{2}=0, \xi_{0}^{2}=0\right)$; (b) the total EE versus $E_{n}^{\mathrm{min}}$ under different algorithms $\left(\varepsilon_{n}^{2}=0.01, v_{n}^{2}=0.01, \xi_{0}^{2}=0.01\right)$

\section{6 结束语}

本文提出了一种基于数能同传的 D2D 网络鲁棒资源分配算法. 具体来讲, 考虑有界信道不确定 性模型, 建立了一个联合优化传输功率、子信道和功率分流因子的能效最大化鲁棒资源分配模型. 针 对该模型, 先采用匹配理论将原问题转化为连续问题, 再使用 Dinkelbach 方法、连续凸近似和变量替 换方法将上述问题转化为凸优化问题, 最后利用拉格朗日对偶理论求得资源分配的解析解. 仿真结果 表明所提算法具有较好的能效和鲁棒性.

\section{参考文献}

1 Agiwal M, Roy A, Saxena N. Next generation 5G wireless networks: a comprehensive survey. IEEE Commun Surv Tut, 2016, 18: 1617-1655

2 Xu Y J, Gui G, Gacanin H, et al. A survey on resource allocation for 5G heterogeneous networks: current research, future trends, and challenges. IEEE Commun Surv Tut, 2021, 23: 668-695

3 Zhou X, Zhang R, Ho C K. Wireless information and power transfer: architecture design and rate-energy tradeoff. IEEE Trans Commun, 2013, 61: 4754-4767

4 Tang J, Shojaeifard A, So D K C, et al. Energy efficiency optimization for CoMP-SWIPT heterogeneous networks. IEEE Trans Commun, 2018, 66: 6368-6383

5 Lu X, Wang P, Niyato D, et al. Wireless charging technologies: fundamentals, standards, and network applications. IEEE Commun Surv Tut, 2016, 18: 1413-1452

6 Sreelakshmy K R, Jacob L. SWIPT techniques in multitier D2D networks for energy efficiency. In: Proceedings of IEEE Region 10 Conference (TEN-CON), 2019. 123-128

7 Khan M S, Jangsher S, Aloqaily M, et al. EPS-TRA: energy efficient peer selection and time switching ratio allocation for SWIPT-enabled D2D communication. IEEE Trans Sustain Comput, 2020, 5: 428-437

8 Shi X Y, Zhang Z W. Multi parameter trade-off of full duplex SWIPT bidirectional DF relay system for D2D Communications. In: Proceedings of IEEE International Conference on Consumer Electronics and Computer Engineering (ICCECE), 2021. 676-682

9 Huang J, Xing C C, Guizani M. Power allocation for D2D communications with SWIPT. IEEE Trans Wirel Commun, 2020, 19: 2308-2320

10 Zhou Z Y, Gao C X, Xu C, et al. Energy-efficient stable matching for resource allocation in energy harvesting-based 
device-to-device communications. IEEE Access, 2017, 5: 15184-15196

11 Yang H H, Ye Y H, Chu X L, et al. Resource and power allocation in SWIPT-enabled device-to-device communications based on a nonlinear energy harvesting model. IEEE Int Things J, 2020, 7: 10813-10825

12 Cheng R, Zhou X T, Zhang H X, et al. Nonorthogonal multiple access in SWIPT enabled cooperative D2D network. In: Proceedings of IEEE/CIC International Conference on Communications (ICCC), 2020. 2377-8644

13 Lim D W, Kang J, Kim H M. Adaptive power control for D2D communications in downlink SWIPT networks with partial CSI. IEEE Wirel Commun Lett, 2019, 8: 1333-1336

14 Xu Y J, Liu Z J, Li G Q, et al. Robust energy efficiency optimization algorithm for NOMA-based D2D communication with simultaneous wireless information and power transfer. J Electr Inf Technol, 2021, 43: 1289-1297 [徐勇军, 刘子 腱, 李国权, 等. 基于 NOMA 的无线携能 D2D 通信鲁棒能效优化算法. 电子与信息学报, 2021, 43: 1289-1297]

$15 \mathrm{Xu} \mathrm{Y} \mathrm{J,} \mathrm{Zhao} \mathrm{X} \mathrm{H,} \mathrm{Liang} \mathrm{Y} \mathrm{C.} \mathrm{Robust} \mathrm{power} \mathrm{control} \mathrm{and} \mathrm{beamforming} \mathrm{in} \mathrm{cognitive} \mathrm{radio} \mathrm{networks:} \mathrm{a} \mathrm{survey.} \mathrm{IEEE}$ Commun Surv Tut, 2015, 17: 1834-1857

16 Xu Y J, Hu R Q, Li G Q. Robust energy-efficient maximization for cognitive NOMA networks under channel uncertainties. IEEE Int Things J, 2020, 7: 8318-8330

17 Ben-Tal A, Nemirovski A. Robust convex optimization. Math Oper Res, 1998, 23: 769-805

18 Papandriopoulos J, Evans J S. SCALE: a low-complexity distributed protocol for spectrum balancing in multiuser DSL networks. IEEE Trans Inform Theory, 2009, 55: 3711-3724

19 Yuan Y L, Yang T, Feng H, et al. An iterative matching-stackelberg game model for channel-power allocation in D2D underlaid cellular networks. IEEE Trans Wirel Commun, 2018, 17: 7456-7471

\title{
Robust resource allocation algorithm in SWIPT-based D2D networks
}

\author{
Yongjun $\mathrm{XU}^{1,2^{*}}$, Meng $\mathrm{YANG}^{1,2}$, Jihua $\mathrm{ZHOU}^{3}$ \& Qianbin $\mathrm{CHEN}^{1,2}$ \\ 1. School of Communication and Information Engineering, Chongqing University of Posts and Telecommunica- \\ tions, Chongqing 400065, China; \\ 2. Chongqing Key Laboratory of Mobile Communications Technology, Chongqing 400065, China; \\ 3. Aerospace New Generation Communications Co., Ltd., Chongqing 401332, China \\ * Corresponding author. E-mail: xuyj@cqupt.edu.cn
}

\begin{abstract}
To improve the operation lifetime of devices and the anti-interference capability of complex electromagnetic environments in device-to-device (D2D) networks, an energy-efficient maximization resource allocation algorithm was proposed for simultaneous wireless information and power transfer (SWIPT)-based D2D networks. Considering the constraints of users' minimum rate, minimum harvested energy, power splitting, and channel allocation, a robust energy-efficient resource allocation model was formulated by jointly optimizing the transmit power, subchannel coefficients, and the power splitting factor. The original NP-hard problem was converted into a deterministic and convex problem by using Dinkelbach's method and the worst-case approach, where the closed-form solutions were obtained by using the matching theory and Lagrange dual theory. Simulation results demonstrated that the proposed algorithm exhibited better robustness and higher energy efficiency.
\end{abstract}

Keywords D2D communications, SWIPT, matching theory, energy efficiency, robust resource allocation 